\title{
Relation of Emittance to Other Optical Properties *
}

J. C. Richmond

(March 4, 1963)

\begin{abstract}
An equation was derived relating the normal spectral emittance of an optically inhomogeneous, partially transmitting coating applied over an opaque substrate to the thickness and optical properties of the coating and the reflectance of the substrate at the coatingsubstrate interface.
\end{abstract}

\section{Introduction}

The $45^{\circ}$ to $0^{\circ}$ luminous daylight reflectance of a composite specimen comprising a partially transparent, spectrally nonselective, light-scattering coating applied to a completely opaque (nontransmitting) substrate, can be computed from the reflectance of the substrate, the thickness of the coating and the reflectivity and coefficient of scatter of the coating material. The equations derived for these conditions have been found by experience [1, 2] ${ }^{1}$ to be of considerable practical usefulness, even though several factors are known to exist in real materials that were not considered in the derivation. For instance, porcelain enamels and glossy paints have significant specular reflectance at the coating-air interface, and no real coating is truly spectrally nonselective.

The optical properties of a material vary with wavelength, but in the derivation of the equations referred to above, they are considered to be independent of wavelength over the rather narrow wavelength band encompassing visible light. The condition of spectral nonselectivity is met sufficiently well by a number of materials to permit use of the pertinent equations with but a single set of optical properties for visible light. Over the enormously wider range of wavelengths within which the emission and absorption of radiant energy are important, few if any materials are sufficiently nonselective to permit use of the pertinent equations with a single set of optical properties applicable to all wavelengths. Hence the optical properties as a function of wavelength are required for computation of wide-range spectral reflectance or emittance of such composite specimens.

The present study was undertaken to derive an equation relating the spectral reflectance (or emittance) of a composite specimen comprised of a partially transmitting, light-scattering coating applied to a completely opaque (nontransmitting) substrate, from the thickness of the coating and the spectral optical properties of the coating and substrate.

\section{Review of the Literature}

The relationship between the thickness and reflectance of a layer of light-scattering materials as a function of the optical constants of the material was developed by Kubelka and Munk [3]. Judd [1] developed graphical methods for the solution of the Kubelka-Munk equation, and demonstrated that it was useful in studying real materials which departed somewhat from the ideal material postulated in development of the equation. Kubelka [4] derived more exact equations in a form that was capable of relatively easy solution. In all of these cases, the materials considered were assumed to be spectrally nonselective, so that a single value of each optical constant could be used throughout the visible range of wavelengths.

Gardon [5] considered the case of an optically homogeneous material with a three-dimensional analysis. Hamaker [6] and Klein [7] worked with powders, which may be considered as a special case of optically inhomogeneous (light-scattering) materials, and derived equations for computing heat transfer by radiation in powders. In these cases a single value applicable to total blackbody radiation was used for each optical property.

\footnotetext{
* This work was sponsored and financed by the George C. Marshall Space Flight Center of NASA.

${ }_{1}^{1}$ Figures in brackets indicate the literature references at the end of this paper.
} 
Saunderson [9] worked with partially transmitting plastics, and used equations for transmittance and reflectance derived by Kubelka and Munk [3], that are similar but not identical to those of Klein [7]. However, he did incorporate a correction for the specular reflection at the plastic-air interface.

\section{Derivation of Equation}

Because of the relationships

$$
A+T+R=1,
$$

in which $A=$ absorptance, $T=$ transmittance, and $R=$ reflectance, and Kirchoff's law,

$$
A=E,
$$

in which $E$ is emittance, ${ }^{2}$ it is possible to compute emittance from reflectance for completely opaque specimens. This method will therefore be used, because of its simplicity as compared to the approach used by Gardon [5].

Consider a flux of completely diffuse radiant energy, incident in uniform geometric distribution over the entire area of an optically flat layer of inhomogeneous isotropic dielectric material having infinite area and uniform thickness. Upon attainment of a steady state condition, the incident flux that is not reflected at the surface will penetrate into the material, diminishing with the depth of penetration, the amount of diminution at any internal plane parallel to the surface being a function only of the distance of the plane from the surface.

Attenuation of the inward-bound diffuse flux will result from absorption of radiant energy within the specimen, and from backscattering by the dispersed particles in the material. The backscattered flux will proceed as completely diffuse flux propagated in a direction normal to the surface outward through the coating. In both cases, the radiant flux lost by lateral scattering will be compensated by an equal gain through similar scattering from adjoining portions of the specimen. The outward-bound flux will also be attenuated by absorption and backscattering. This backscattered flux will reinforce the incoming flux.

Under the postulated conditions, a one-dimensional mathematical analysis can describe the variation in diffuse radiant flux density along a line normal to the surface, due to absorption and scattering within the material, as a function of distance from the coating-substrate interface.

In using the one-dimensional analysis, the diffuse radiant flux traversing unit area normal to the direction of propagation is considered as being made up of two directionally opposed portions, one $I$, outward from the interior of the specimen and normal to the flat surface, and the other $J$, in the opposite inward direction. A spectral absorption coefficient, $K$, is defined by equating $K I d x$ to the reduction in $I$ by absorption within a layer of infinitesimal thickness, $d x$. A scattering coefficient, $S$, is similarly defined by equating $S I d x$ to the flux scattered backwards from $I$ in the unidirectional beam (and therefore included in $J$ ) within a layer of infinitesimal thickness, $d x$.

Within the distance $d x$ the flux, $I$, will be not only diminished by absorption and scattering, but also augmented by the backscattering from $J$. Hence we can write

$$
\begin{gathered}
d I / d x=-(K+S) I+S J \\
d J / d x=(K+S) J-S I .
\end{gathered}
$$

These are the general differential equations first used by Kubelka and Munk [3] as a starting point, and subsequently by many other investigators.

\footnotetext{
2 Emittance, as used in this paper, is defined as follows: Emittance is a property of a specimen; it is the ratio of its emissive power to that of a blackbody radiator at the same temperature and under the same spectral and geometric conditions of viewing.

Emissive power is the rate of thermal emission expressed as radiant flux per unit surface area.
} 

are

Hamaker's [6] solutions for eqs (3) and (4), considering both absorption and scattering,

$$
\begin{aligned}
& I=L_{1}(1-\beta) e^{\sigma x}+L_{2}(1+\beta) e^{-\sigma x} \\
& J=L_{1}(1+\beta) e^{\sigma x}+L_{2}(1-\beta) e^{-\sigma x}
\end{aligned}
$$

where $L_{1}$ and $L_{2}$ are constants determined by the boundary conditions, and where

and

$$
\sigma=\sqrt{K(K+2 S)}
$$

$$
\beta=\sqrt{K /(K+2 S)} .
$$

\subsection{Equations Applicable to Coatings}

Most coatings, such as paint, porcelain enamel, and ceramic coatings, unlike the materials postulated by Kubelka [4] and dealt with by Hamaker [6] and Klein [7], have a disperse medium other than air, usually of a glassy nature, in which the scattering particles are distributed. Hence specular reflectance at the coating-air and coating-substrate interfaces must be considered. This structural difference will give different boundary conditions than were considered by previous authors.

In the systems under consideration, the reflectance at the interfaces will be considered, as follows:

$\rho_{e}=$ specular reflectance at the coating-air interface for externally incident, completely diffuse radiant flux $\left(J_{e}\right)$.

$\rho_{s}=$ reflectance of the substrate for completely diffuse radiant flux $\left(J_{0}\right)$ incident upon the substrate from the coating.

$\rho_{i}=$ specular reflectance of the coating-air interface for completely diffuse radiant flux $\left(I_{D}\right)$ incident from within the coating.

Let $x$ be the perpendicular distance from the coating-substrate interface to a point in the coating, and $D$ be the thickness of the coating.

If only the disposition of incident flux be considered, and if radiant energy emitted by the specimen be ignored, the boundary conditions for substitution in eqs (5) and (6) are

$$
\begin{gathered}
I=J_{o} \rho_{s} \text { at } x=0 \\
J=J_{e}\left(1-\rho_{e}\right)+I_{D} \rho_{i} \text { at } x=D
\end{gathered}
$$

when $x=0, e^{\sigma x}=e^{-\sigma x}=1$, hence from eqs (5), (6), and (9)

$$
L_{1}(1-\beta)+L_{2}(1+\beta)=\rho_{s}\left[L_{1}(1+\beta)+L_{2}(1-\beta)\right],
$$

and from eqs (5), (6), and (10),

$$
L_{1}(1+\beta) e^{\sigma D}+L_{2}(1-\beta) e^{-\sigma D}=J_{e}\left(1-\rho_{e}\right)+\rho_{i}\left[L_{1}(1-\beta) e^{\sigma D}+L_{2}(1+\beta) e^{-\sigma D}\right] .
$$

\footnotetext{
${ }^{3}$ It may be worth while to mention the physical significance of $\sigma$ and $\beta$. As can be seen in eq (7), $\sigma$ is a type of extinction coefficient. If $S=0$, $\sigma=K$; if $K=0, \sigma=0$. The combination $\sigma x$ is a dimensionless optical thickness; all layers having the same $\sigma x$ product will produce the same attenuation in a beam of radiant energy. $\sigma$ is determined by the absolute values of $S$ and $K$. $\beta$, on the other hand, is a function of the relative magnitudes of $S$ and $K$. If $S=0, \beta=1$, and if $K=0, \beta=0 . \beta$ is shown by Klein [7] to be related to emissivity, $E_{\infty}$, and reflectivity, $R_{\infty}$, by the equations

$$
E_{\infty}=\frac{2 \beta}{1+\beta} \text { and } R_{\infty}=\frac{1-\beta}{1+\beta},
$$

provided there is no specular reflection at the surface. For glossy materials, these relationships are modified by specular reflection at the surface.
} 
Solving eqs (11) and (12) for $L_{1}$ and $L_{2}$, we get:

$$
\begin{gathered}
L_{1}=\frac{J_{e}\left(1-\rho_{e}\right)\left[(1+\beta)-\rho_{s}(1-\beta)\right]}{\left[(1+\beta)-\rho_{i}(1-\beta)\right]\left[(1+\beta)-\rho_{s}(1-\beta)\right] e^{\sigma D}-\left[(1-\beta)-\rho_{i}(1+\beta)\right]\left[(1-\beta)-\rho_{s}(1+\beta)\right] e^{-\sigma D}} \\
L_{2}=\frac{-J_{e}\left(1-\rho_{e}\right)\left[(1-\beta)-\rho_{s}(1+\beta)\right]}{\left[(1+\beta)-\rho_{i}(1-\beta)\right]\left[(1+\beta)-\rho_{s}(1-\beta)\right] e^{\sigma D}-\left[(1-\beta)-\rho_{i}(1+\beta)\right]\left[(1-\beta)-\rho_{s}(1+\beta)\right] e^{-\sigma D}} .
\end{gathered}
$$

The equations can be simplified by making the following substitutions:

Let

$$
\begin{aligned}
M & =(1+\beta)-\rho_{s}(1-\beta) \\
N & =(1+\beta)-\rho_{i}(1-\beta) \\
O & =(1-\beta)-\rho_{s}(1+\beta) \\
P & =(1-\beta)-\rho_{i}(1+\beta) .
\end{aligned}
$$

Then eqs (13) and (14) become

$$
\begin{aligned}
& L_{1}=\frac{J_{e}\left(1-\rho_{e}\right) M}{M N e^{\sigma D}-O P e^{-\sigma D}} \\
& L_{2}=\frac{-J_{e}\left(1-\rho_{e}\right) O}{M N e^{\sigma D}-O P e^{-\sigma D}} .
\end{aligned}
$$

The overall specular plus diffuse reflectance, $R$, of the specimen is defined as the reflected flux divided by the incident flux. The reflected flux will include the fraction of $J_{e}$ that is specularly reflected at the coating-air interface. That is

$$
R=\frac{J_{e} \rho_{e}+\left(1-\rho_{i}\right) I_{D}}{J_{e}}
$$

From eqs (5), (19), and (20),

$$
I_{D}=\frac{J_{e}\left(1-\rho_{e}\right)\left[(1-\beta) M e^{\sigma D}-(1+\beta) O e^{-\sigma D}\right]}{M N e^{\sigma D}-O P e^{-\sigma D}},
$$

hence

$$
R=\rho_{e}+\left(1-\rho_{e}\right)\left(1-\rho_{i}\right) \frac{(1-\beta) M e^{\sigma D}-(1+\beta) O e^{-\sigma D}}{M N e^{\sigma D}-O P e^{-\sigma D}} .
$$

$R$ in eq (23) is the reflectance of the composite specimen under conditions of completely diffuse illumination and hemispherical viewing. The equation has been written in terms of the coating parameters $\beta, \sigma, \rho_{e}, \rho_{i}, \rho_{s}$, and the thickness of the coating, $D$. The values of these parameters vary with wavelength. The equation applies only to spectral reflectance, $R_{\lambda}$, under the given geometric conditions, and spectral values of the coating and coatingsubstrate parameters, $\beta_{\lambda}, \sigma_{\lambda}, \rho_{e \lambda}, \rho_{i \lambda}$, and $\rho_{s \lambda}$ are required. The subscript $\lambda$ indicates that the symbol applies to the spectral value at wavelength $\lambda$.

When transmittance is zero, as it is in the case of the coated specimens under consideration, the emittance is equal to one minus the reflectance, as is indicated by eqs (1) and (2), if consistent geometric conditions of incident, reflected, and emitted flux are used. $R$ has been defined as the spectral reflectance under conditions of diffuse illumination and hemispherical viewing. The emittance corresponding to $R$ is $E_{H}$, the hemispherical spectral emittance. Hence we can write

$$
E_{H}=1-\left\{\rho_{e}+\left(1-\rho_{e}\right)\left(1-\rho_{i}\right) \frac{(1-\beta) M e^{\sigma D}-(1+\beta) O e^{-\sigma D}}{M N e^{\sigma D}-O P e^{-\sigma D}}\right\} .
$$


It is legitimate also to apply eqs (1) and (2) to evaluate the normal spectral emittance, $E_{N}$, if, and only if, the geometric conditions of irradiation and viewing under which the external reflectance, $\rho_{e}$, is measured are (1) normal incidence and hemispherical viewing or (2) perfectly diffuse incidence and normal viewing.

If we consider that the internal reflectance of the coating plus substrate, neglecting the effect of specular reflectance at the coating-air interface, is $R_{i}$, then with diffuse illumination and hemispherical viewing

$$
R=\rho_{e}+\left(1-\rho_{e}\right) R_{1}\left(1-\rho_{i}\right) .
$$

Equation (25) is another way of writing eq (24), with $R_{i}$ replacing the fraction containing exponential terms.

$$
R_{i}=\frac{(1-\beta) M e^{\sigma D}+(1+\beta) O e^{-\sigma D}}{M N e^{\sigma D}-O P e^{-\sigma D}} .
$$

For normal illumination and hemispherical viewing, the equation becomes

$$
R_{N}=\rho_{N}+\left(1-\rho_{N}\right) R_{i}\left(1-\rho_{i}\right)
$$

in which $R_{i}$ is again the internal reflectance, neglecting the effect of specular reflectance at the coating-air interface, of the coating plus substrate. If $R_{i}$ is the same for normal and diffuse incident flux, ${ }^{4}$ we can write

$$
E_{N}=1-\left\{\rho_{N}+\left(1-\rho_{N}\right)\left(1-\rho_{i}\right) \frac{(1-\beta) M e^{\sigma D}-(1+\beta) O e^{-\sigma D}}{M N e^{\sigma D}-O P e^{-\sigma D}}\right\}
$$

which is the desired equation.

Substituting for $M, N, O$, and $P$ from eqs (15), (16), (17), and (18), and making use of the relationship

and

$$
2 \sinh x=e^{x}-e^{-x}
$$

eq (28) reduces to

$$
2 \cosh x=e^{x}+e^{-x}
$$

$$
E_{N}=\left(1-\rho_{N}\right)\left\{1-\left(1-\rho_{i}\right) \frac{\left[\left(1-\rho_{s}\right)-\beta^{2}\left(1+\rho_{s}\right)\right] \sinh \sigma D+2 \rho_{s} \beta \cosh \sigma D}{\left[\left(1+\beta^{2}\right)\left(1+\rho_{i} \rho_{s}\right)-\left(1-\beta^{2}\right)\left(\rho_{i}+\rho_{s}\right)\right] \sinh \sigma D+2 \beta\left(1-\rho_{i} \rho_{s}\right) \cosh \sigma D}\right\} .
$$

The specular reflectances at the coating-air interface, $\rho_{e}$ and $\rho_{i}$, are for diffuse illumination and hemispherical viewing. The value to be used for $\rho_{s}$ must be determined experimentally, because the coating-substrate interface is normally rough, and the reflectance may be affected by interaction between coating and substrate at the interface. For an optically smooth interface, which may be approximated by the coating-air interface for some coatings at some wavelengths, $\rho_{e}$ and $\rho_{i}$ can be computed from the index of refraction of the coating ${ }^{5}$ by integration of the Fresnel equation over a hemisphere. In its general form, the equation for dielectrics can be written

$$
2 \rho_{d}=\frac{\sin ^{2}(\varphi-\theta)}{\sin ^{2}(\varphi+\theta)}+\frac{\tan ^{2}(\varphi-\theta)}{\tan ^{2}(\varphi+\theta)}
$$

in which $\rho_{d}$ is the directional specular reflectance for unpolarized parallel flux, $\phi$ is the angle of incidence, and $\theta$ is the angle of refraction, both angles measured from the normal to the interface. The angles $\phi$ and $\theta$ are related to index of refraction, $n$, by Snell's law. In its most general form, this is $n_{1} \sin \phi=n_{2} \sin \theta$, in which $n_{1}$ is the index of refraction of the medium on the

\footnotetext{
${ }^{4}$ This condition will be approximated if the incident flux is completely diffused after traversing a small thickness of the coating.

5 Most optically inhomogeneous coatings consist of opacifying particles dispersed in a continuous medium or vehicle. The scattering is due to the difference in indices of refraction of the opacifier and vehicle. For glossy coatings, the opacifying particles are not usually exposed at the surface of the coating, and the effective index of refraction for specular reflection is that of the vehicle.
} 
side of the interface from which the flux is incident, and $n_{2}$ is that on the opposite side of the interface. In the case of flux incident from vacuum, $n_{1}=1$. In the case of air, no serious error is introduced by considering $n_{1}=1 \quad\left(n_{1}=1.0003\right.$, approximately, for air under normal conditions).

The reflectance of an optically smooth surface of index of refraction $n$, for completely diffuse flux incident from vacuum (or air) can be expressed as

$$
\rho_{e}=\frac{\int_{0}^{\pi / 2} \sin \varphi \cos \varphi\left\{\frac{\sin ^{2}(\varphi-\theta)}{\sin ^{2}(\varphi+\theta)}+\frac{\tan ^{2}(\varphi-\theta)}{\tan ^{2}(\varphi+\theta)}\right\} d \varphi}{2 \int_{0}^{\pi / 2} \sin \varphi \cos \varphi d \varphi}
$$

This expression has been integrated by Walsh [8] to give

$$
\begin{aligned}
\rho_{e}=\frac{1}{2}+\frac{(n-1)(3 n-1)}{6(n+1)^{2}}+\left\{\frac{n^{2}\left(n^{2}-1\right)^{2}}{\left(n^{2}+1\right)^{3}}\right\} \ln \frac{(n-1)}{(n+1)} & \\
& \quad-2 n^{3} \frac{\left(n^{2}+2 n-1\right)}{\left(n^{2}+1\right)\left(n^{4}-1\right)}+\left\{\frac{8 n^{4}\left(n^{4}+1\right)}{\left(n^{2}+1\right)\left(n^{4}-1\right)^{2}}\right\} \ln n .
\end{aligned}
$$

For completely diffuse flux incident on the interface from within the material of index $n$, all of the radiant energy that is incident at angles greater than the critical angle, $\varphi_{c}$, will be totally reflected. The critical angle is that at which $\theta=\pi / 2$, or $\sin \varphi_{c}=\frac{1}{n}$. The fraction of flux incident at angles from $\sin ^{-1} \frac{1}{n}$ to $\pi / 2$ can be obtained by integration and divided by the total flux to give the fraction $1-\frac{1}{n^{2}}$ of the incident flux that is totally reflected. Hence only $\frac{1}{n^{2}}$ of the total flux will be incident at angles at which it is refracted, and a fraction $\rho_{e}$ of that flux will be internally reflected, as indicated in eq (33). Hence we can write

$$
\rho_{i}=1-\frac{1}{n^{2}}+\frac{\rho_{e}}{n^{2}} \quad \text { or } \quad n^{2}\left(1-\rho_{i}\right)=\left(1-\rho_{e}\right)
$$

The expression for $\rho_{e}$ given in eq (34) is somewhat complex. Judd [10] gives computed values of $\rho_{e}$ and $\rho_{i}$ for indices of refraction from 1.00 to 1.60 in increments of 0.01 .

The geometric distribution of the emitted flux will be determined by the directional reflectance at the coating-air interface, and can be computed from the refractive index by means of the Fresnel equation. For normally incident parallel flux, the Fresnel equation reduces to

and

$$
\rho_{N}=\left(\frac{n-1}{n+1}\right)^{2}
$$

$$
1-\rho_{N}=\frac{4 n}{(n+1)^{2}}
$$

where $\rho_{N}$ is specular reflectance for normally incident flux.

The normal spectral emittance, $E_{N}$, of any specimen can be computed from the hemispherical spectral emittance by use of the Fresnel equations for reflectance of internally incident normal and diffuse flux. This conversion is not affected by the coefficient of scatter of the coating.

\section{Discussion}

In the case of paper, layers of powder and unglazed porous ceramic materials, air may be considered the continuous phase, and there will be no specular reflection at the interfaces. If, in addition, there is no substrate, or if the reflectance of the substrate is zero, the conditions will 
be identical to those postulated by Klein [7]. If zero is substituted for $\rho_{e}, \rho_{s}$, and $\rho_{i}$ in eq (23), it reduces to

$$
R=\frac{\left(1-\beta^{2}\right) \sinh \sigma D}{\left(1+\beta^{2}\right) \sinh \sigma D+2 \beta \cosh \sigma D},
$$

which is identical to Klein's [7] eq (46).

\subsection{Properties of Nonabsorbing, Nonscattering Layers}

It may be of interest to consider how the emittance and reflectance of a coating will vary as $S$ and $K$ approach zero. Obviously, if both $S$ and $K$ are zero, the material will have perfect transmittance, and both reflectance and absorptance (or emittance) will be zero. This is true for a perfect vacuum, and is closely approximated by a gas at those wavelengths at which no absorption occurs.

$$
\text { a. Properties of Nonscattering Layers }
$$

If there is no scattering, $S=0$, and eqs (3) and (4) become

and

$$
d I / d x=-K I
$$

hence

$$
d J / d x=K J
$$

$$
I_{(x+\Delta x)}=I_{x} e^{-K \Delta x},
$$

where $I_{x}$ represents the flux density at a level $x$ within the material, and $I_{(x+\Delta x)}$ represents the flux density after traversing a thickness $\Delta x$ of the material. Equation (41) differs from the familiar Bouguer's law only in that the attenuation of completely diffuse flux is twice as rapid as that of unidirectional flux.

If the scattering coefficient, $S$, is zero, which will be approached by optically homogeneous materials, such as optical glass and many single crystals, then $\sigma=K$ and $\beta=1$, hence (24) reduces to

$$
E_{H}=\left(1-\rho_{e}\right)\left\{1-\frac{\left(1-\rho_{i}\right) \rho_{s} e^{-K D}}{e^{K D}-\rho_{i} \rho_{s} e^{-K D}}\right\}
$$

However, when $S$ becomes small, the internally reflected flux will not be rediffused, if reflected from optically smooth surfaces. Thus (42) is only valid if the coating-substrate interface is so rough that perfectly diffuse reflection occurs. For the more general case, where this interface approaches optical smoothness, a three-dimensional analysis of the type used by Gardon [5] is more nearly valid.

\section{b. Properties of Nonabsorbing Layers}

If the absorption coefficient, $K$, is zero, as will be approximated at some wavelengths by a freshly smoked layer of magnesium oxide or by freshly fallen snow, both $\sigma$ and $\beta$ become zero, and eq (24) becomes indeterminate, which would be expected for an opaque coating of the specified materials, but not for a composite specimen. However, under these conditions, eqs (3) and (4) become

$$
d I / d x=-S(J-I)=d J / d x .
$$

This equation can be solved by a procedure similar to those used for eqs (3) and (4) to give

$$
\begin{gathered}
I=\frac{J_{e}\left[S x\left(1-\rho_{e}\right)\left(1-\rho_{s}\right)+\rho_{s}\left(1-\rho_{e}\right)\right]}{S D\left(1-\rho_{i}\right)\left(1-\rho_{s}\right)+1-\rho_{i} \rho_{s}} \\
J=\frac{J_{e}\left[S x\left(1-\rho_{e}\right)\left(1-\rho_{s}\right)+\left(1-\rho_{e}\right)\right]}{S D\left(1-\rho_{i}\right)\left(1-\rho_{s}\right)+1-\rho_{i} \rho_{s}} .
\end{gathered}
$$


Substituting eq (44) in eq (21) we get

$$
R=\rho_{e}+\left(1-\rho_{i}\right)\left(1-\rho_{e}\right) \frac{S D\left(1-\rho_{s}\right)+\rho_{s}}{S D\left(1-\rho_{i}\right)\left(1-\rho_{s}\right)+\left(1-\rho_{i} \rho_{s}\right)} \cdot
$$

If $\rho_{e}, \rho_{i}$, and $\rho_{s}$ are zero, as they were under the conditions postulated by Klein [7], eq (46) reduces to

$$
R=\frac{S D}{S D+1}
$$

which is identical to Klein's eq (48).

Under the conditions postulated for the derivation of eq (47), the emittance will approach zero and the reflectance will approach one as the coating approaches a thickness at which it is completely opaque, which will only occur at infinite thickness. Since $K=0$, the absorptance is zero by definition, and the transmittance is one minus the reflectance. Hence we can write

$$
T=\frac{1}{S D+1}
$$

which is identical to Klein's eq (a7).

\subsection{Example of Computed Emittance}

Figure 1 is a plot of emittance, computed from eq (31), as a function of thickness for composite specimens with optically smooth surfaces comprised of a coating having an index of refraction of 1.40 (from which $\rho_{e}=0.028, \rho_{i}=0.53$ ), a value of $\beta$ of 0.8 , and values of $\sigma$ of 40 , $20,13.3$, and $10.0 \mathrm{~mm}^{-1}$, respectively, applied over a mat substrate having a reflectance into the coating of 0.9 . (The values of $S$ and $K$ corresponding to a $\beta$ of 0.8 and $\sigma$ of $40,20,13.3$, and $10 \mathrm{~mm}^{-1}$ are as follows: $S=8.0,4.0,2.65$, and $2.0 \mathrm{~mm}^{-1}$ and $K=28.44,14.22,9.42$, and $7.11 \mathrm{~mm}^{-1}$, respectively.) These conditions might be approximated at wavelengths in the visible by a glossy gray paint applied over a white backing. All of the coating materials have the same emissivity (about 0.918), but the coating with a $\sigma$ value of $40 \mathrm{~mm}^{-1}$ reaches 99 percent of this value at a thickness of $0.05 \mathrm{~mm}$, while the coating with a $\sigma$ value of $10 \mathrm{~mm}^{-1}$ requires a thickness of $0.2 \mathrm{~mm}$ to reach the same value.

The plotted emittance at zero thickness of the coating is greater than the emittance (0.10) of the substrate because the coating has a lower index of refraction than the substrate, and hence reduces reflection at the interface. The plotted value is what would be obtained with a very thin, perfectly transparent glossy coating having an index of refraction of 1.40.

Figure 1. Computed normal spectral emittance, plotted as a function of coating thickness, for a composite specimen comprised of partially transmitting coatings with optically smooth surfaces having an index of refraction of $1.40, \beta$ value of 0.80 , and $\sigma$ values of $40,20,13.3$, and $10 \mathrm{~mm}^{-1}$, respectively, applied over a substrate having a reflectance of 0.90 , plotted as a function of coating thickness. These conditions would be approximated by a glossy gray paint applied over a polished metal or white substrate.

Note that the emittance at zero coating thickness is not that of the substrate. It was assumed that even at zero thickness, there would be ane. It was assumed that even at zero thickness, there ally, such effect would be produced by a transparent coating of index of refraction of 1.40 . The coating having a $\sigma$ value of index of refraction of 1.40 . The coating having a $\sigma$ value of
$40 \mathrm{~mm}^{-1}$ becomes essentially opaque at about $0.05 \mathrm{~mm}$, and the other coatings at successively greater thicknesses.

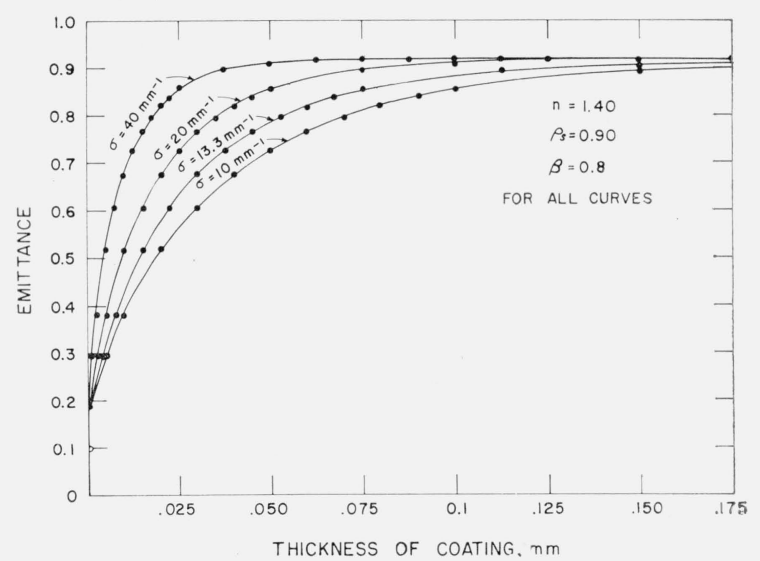




\subsection{Limitations to Equations}

There are a number of conditions encountered in practice that deviate from the conditions postulated in the derivation of eq (24). There will always be a thermal gradient normal to the surface in any situation where a body is heated internally and is dissipating radiant energy, and the effect of such a gradient has been omitted in the derivation. In the case of thick ceramic coatings (typically $0.5 \mathrm{~mm}$ or more), significant errors may be introduced by ignoring the effect of thermal gradients, especially at high temperatures. The gradients will be negligibly small when the specimen and surroundings are at room temperature or below, and the effect of the gradients in thin coatings, typically less than $0.1 \mathrm{~mm}$, is considered to be negligibly small even at high temperatures.

During firing of porcelain enamels and some other types of ceramic coatings, on some metals, there is appreciable chemical reaction at the coating-metal interface, and some of the reaction products diffuse into the coating for measurable distance. The reaction products may change the reflectance of the substrate into the coating, and the reaction products diffusing into the coating may significantly change its optical properties near the interface. The extent and importance of these effects depend upon the particular materials involved, their thicknesses, the firing, and the service conditions. When the total effect of these factors is large, appropriate adjustments in the values substituted into the equation are required for its useful application. Most types of organic and flame-sprayed ceramic coatings, however, will be essentially free from these effects.

In the case of some paints, there may be segregation of the pigment particles within the vehicle during drying, which will also tend to invalidate the equation. For mat coatings, where the coating-air interface is not optically smooth even on a micro scale, it may be necessary to measure $\rho_{e}$ and $\rho_{i}$ experimentally, instead of computing them from the index of refraction.

\section{Summary}

The thermal radiation properties of a composite specimen, comprised of a partially transmitting coating applied over an opaque substrate, usually vary significantly with the thickness of the coating. An equation was derived relating these properties to the thickness of the coating, the reflectance of the substrate, and the optical properties of the coating material. If the optical properties of a coating and the reflectance of the substrate are known as functions of wavelength, the equation can be used to compute (1) the normal spectral emittance (or reflectance) of any thickness of coating over the substrate or (2) the thickness of the coating over the substrate required to give any normal spectral emittance (or reflectance) within any given wavelength interval intermediate between the emittance of the substrate and of an infinitely thick coating.

The author gratefully acknowledges the assistance of Louis Joseph, of the Applied Mathematics Division, in checking the mathematics, and of Deane B. Judd, of the Optics and Metrology Division, for his advice and helpful suggestions.

\section{List of Symbols}

$A=$ absorptance.

$T=$ transmittance.

$R=$ reflectance.

$E=$ emittance.

$I=$ diffuse radiant flux proceeding outward from the interior of a specimen.

$J=$ diffuse radiant flux proceeding inward toward the interior of a specimen.

$K=$ absorption coefficient. 
$S=$ backscattering coefficient.

$x=$ distance from the coating-substrate interface to a point in the coating.

$L=$ a constant that depends upon boundary conditions.

$\sigma=\sqrt{K(K+2 S)}$.

$\beta=\sqrt{K /(K+2 S)}$.

$\rho_{e}=$ reflectance of the coating-air interface for externally-incident diffuse radiant flux.

$\rho_{s}=$ reflectance of the substrate for diffuse radiant flux incident on the coating-substrate interface from within the coating.

$\rho_{i}=$ reflectance of the coating-air interface for internally incident diffuse radiant flux.

$J_{e}=$ externally incident diffuse radiant flux.

$J_{0}=$ flux $J$ at $x=0$ (at coating-substrate interface).

$I_{D}=$ flux $I$ at $x=D$ (at coating-air interface).

$D=$ thickness of coating.

$M=(1+\beta)-\rho_{s}(1-\beta)$.

$N=(1+\beta)-\rho_{i}(1-\beta)$.

$O=(1-\beta)-\rho_{s}(1+\beta)$.

$P=(1-\beta)-\rho_{i}(1-\beta)$.

$n=$ index of refraction.

$n_{1}=$ index of refraction in medium from which flux is incident.

$n_{2}=$ index of refraction in medium into which flux is refracted.

$\varphi=$ angle of incidence, from the normal.

$\theta=$ angle of refraction, from the normal.

$\varphi_{c}=$ critical angle of incidence for total internal reflectance.

$E_{H}=$ hemispherical spectral emittance.

$\rho_{N}=$ reflectance of the coating-air interface for normally incident parallel flux.

$E_{N}=$ normal spectral emittance.

$R_{i}=$ internal reflectance of a coating.

\section{References}

[1] Judd, D. B., Optical specification of light-scattering materials, J. Res. NBS 19, 287 (1937) RP1026.

[2] ASTM Method C 347-57, Reflectivity and Coefficient of Scatter of White Porcelain Enamels, 1961 ASTM Book of Standards, pp. 612-615.

[3] Kubelka, P., and F. Munk, Ein Beitrag zur Optik der Farbanstriche, Z. tech. Physik, 12, 593 (1931).

[4] Kubelka, P., New contributions to the optics of intensely light-scattering materials, Part I. J. Opt. Soc. Am. 38, 448 (1948).

[5] Gardon, R., The emissivity of transparent materials, J. Am. Ceram. Soc. 39 [8], 278-285 (1956).

[6] Hamaker, H. C., Radiation and heat conduction in light-scattering material, Philips Res. Reports, 2, 55-67, 103-111, 112-125, 420-425 (1947).

[7] Klein, J. D., Heat transfer by radiation in powders, Doctor's Dissertation, Mass. Inst. of Technology (1960).

[8] Walsh, J. W. T., The reflection factor of a polished glass surface for diffused light, Dept. Sci. and Ind. Res. Illumination Research, Tech. Paper No. 2, p. 10 (1926).

[9] Saunderson, J. L., Calculation of the color of pigmented plastics, J. Opt. Soc. Am. 32 [12], 727-736.

[10] Judd, D. B., Fresnel reflection of diffusely incident light, J. Research NBS 29, 329-332 (1942) RP1504.

(Paper 67C3-132) 